\title{
Clitoral Epidermoid Cyst Presenting as Pseudoclitoromegaly of Pregnancy
}

jing W. Hughes, MD, PhD ${ }^{1}$ Marsha K. Guess, MD ${ }^{2}$ Adam Hittelman, MD, $\mathrm{PhD}^{3}$ Sallis Yip, MD John Astle, MD, PhD ${ }^{5}$ Lubna Pal, MBBS, MRCOG ${ }^{2}$ Silvio E. Inzucchi, MD ${ }^{1}$ Antonette T. Dulay, MD

${ }^{1}$ Section of Endocrinology and Metabolism, Yale University School of Medicine, New Haven, Connecticut

2 Department of Obstetrics, Gynecology and Reproductive Sciences, Address for correspondence Jing W. Hughes, MD, PhD, 333 Cedar Street, FMP 105, New Haven, CT 06519 (e-mail: jing.hughes@yale.edu).

Yale University School of Medicine, New Haven, Connecticut

${ }^{3}$ Department of Urology and Pediatric Urology, Yale University School of Medicine, New Haven, Connecticut

${ }^{4}$ Department of Urogynecology and Reconstructive Pelvic Surgery, Yale University School of Medicine, New Haven, Connecticut

${ }^{5}$ Department of Pathology, Yale University School of Medicine, New Haven, Connecticut

6 Yale Maternal-Fetal Medicine, Department of Obstetrics, Gynecology and Reproductive Sciences, Yale University School of Medicine, New Haven, Connecticut

\begin{abstract}
Keywords

- clitoromegaly

- pseudoclitoromegaly

- pregnancy

- epidermoid cyst

Objective Acquired clitoromegaly is rare and may result from hormonal and nonhormonal causes, and evaluation of the pregnant patient with clitoromegaly invokes a specific set of differential diagnoses.

Methods Case report.

Results We describe the case of a young woman with pregnancy-associated clitoral enlargement whose hormonal evaluation proved negative. Further investigation concluded that an epidermoid cyst was the culprit of her pseudoclitoromegaly. The patient underwent successful surgical resection and has had no recurrence at her subsequent pregnancy.

Conclusion We review the differential diagnosis of clitoromegaly, including hormonal and nonhormonal causes, with focus on the evaluation of pregnancy-associated clitoromegaly.
\end{abstract}

\section{Case Report}

A 31-year-old gravida 2, para 1 presented at 9 weeks' gestation with concerns relating to noticeable clitoral enlargement. A review of her gynecologic history revealed normal menarche at age 12 and normal breast and genital development during puberty. She denied any history of menstrual irregularities, excessive facial or body hair, acne, scalp hair loss, change in the voice timbre, regression in breast size, or difficulty conceiving. Patient also denied any genital trauma, dyspareunia, pruritus, or discharge. Family history was non-

received

December 4, 2012

accepted after revision

January 2, 2013

published online

February 7, 2013

contributory with no consanguinity. Two years prior, she became pregnant with her first child and, starting at 20 weeks' gestation, noticed gradual painless clitoral enlargement until it reached a documented size of $5 \times 2 \mathrm{~cm}$. The patient was otherwise asymptomatic and did not undergo any further evaluation. Within 2 months of delivering a healthy male infant, the patient experienced spontaneous regression in clitoral size to its prepregnancy baseline. However, at 5 months postpartum, her clitoris began to grow again and remained enlarged through spring 2012 when she became pregnant for the second time.

Copyright $\odot 2013$ by Thieme Medical Publishers, Inc., 333 Seventh Avenue, New York, NY 10001, USA. Tel: +1(212) 584-4662.
DOI http://dx.doi.org/ 10.1055/s-0033-1334461. ISSN 2157-6998. 
Psychological distress relating to the persistently enlarged clitoris in her second pregnancy finally prompted consultation to Endocrinology and Maternal-Fetal Medicine. A transvaginal pelvic ultrasound done at 6 weeks' gestation showed unremarkable ovaries and a normal intrauterine gestation whose crown-rump length was consistent with established dates. On physical exam, the patient did not exhibit any systemic signs of virilization. Her voice was normal female, the amount and pattern of body hair distribution were gender-appropriate, and there was no evidence of acne, temporal balding, or abnormal muscular development. Examination of her external genitalia identified a markedly enlarged clitoris, measuring $7 \times 2 \mathrm{~cm}$, with the size and appearance resembling that of a microphallus (-Fig. 1). The clitoral hood was minimally retractable, and the clitoral body was firm and demonstrated some mobility along the lateral horizontal plane. A soft fluctuance approximately $1 \times 1 \mathrm{~cm}$ was palpated at the base. There was no erythema or tenderness. The introitus was unremarkable, and the labia majora and minora appeared normal. A single urethral meatus was clearly visualized in the normal anatomical location. Speculum exam revealed a well-estrogenized vagina and a normal-appearing cervix, and bimanual exam was consistent with early pregnancy. Notably, there was no communication between the anterior vaginal wall and the clitoris.

Given the striking appearance of the clitoris, a spectrum of possible diagnoses was considered, including acquired virilization syndromes of pregnancy, hermaphroditism, adrenal hyperplasia, ovarian or adrenal neoplasms, exogenous androgen exposure, and various forms of pseudoclitoromegaly, which are explored in further detail in the Discussion section of this report. Importantly, prophylactic dexamethasone therapy was considered early in the patient's evaluation given the possibility of late-onset congenital adrenal hyperplasia and its associated virilizing risk for a female fetus. ${ }^{1}$ This approach was deferred until further assessment, given the lack of maternal virilization. We next performed an extensive evaluation of the pituitary-adrenal-ovarian hormonal axes. These studies revealed gestation-appropriate levels of serum adrenocorticotropic hormone, morning cortisol, sex hormone binding globulin, dehydroepiandrosterone sulfate, androstenedione, total and free testosterone, 17-hydroxypregnenolone, 17-hydroxyprogestoerone, and 11-deoxycortisol (- Table 1).

Unfortunately, this pregnancy resulted in a spontaneous miscarriage at 11 weeks' gestation. Pelvic magnetic resonance imaging was performed just after the miscarriage for better elucidation of the clitoral anatomy. This revealed a $6.2 \times 4.2-\mathrm{cm}$ midline cystic mass extending from the bladder base and neck into the anterior periurethral space (-Fig. 2). Echotexture of the cyst contents was consistent with proteinaceous material that demonstrated marked T1 hyperintensity and mild T2 hyperintensity (-Fig. 2B). Based on the magnetic resonance findings, we suspected a possible congenital urethral duplication cyst or atypical obstructed urethral diverticulum.

The patient was referred for urogynecological evaluation and subsequently underwent resection of the clitoral mass. The mass was contained by an easily identifiable cyst wall, which was meticulously dissected away from the underlying crura. Curiously, the lesion was so large that it extended atop the anterior bladder wall, though intraoperative cystogram and cystourethroscopy revealed no abnormal drainage from the structure to surrounding lumens including the urethra and bladder. Histopathologic examination of the patient's surgical specimen revealed a $7.5 \times 2.0 \times 0.4 \mathrm{~cm}$ deflated unilocular cyst, containing a hemorrhagic cloudy fluid, with a wall thickness averaging $0.1 \mathrm{~cm}$ and a slightly granular lining. Microscopically, the specimen was marked by a squamous epithelium ( $\boldsymbol{\sim}$ Fig. $\mathbf{3 A}$ ), and the interior was composed of extensive histiocyte infiltrates, specifically hemosiderinladen macrophages amid necrotic debris. These findings were thought characteristic of a ruptured epidermal inclusion cyst ( - Fig. 3B). To exclude the presence of endometrial stroma or urothelial tissue, $^{2}$ the specimen was submitted for CD10 and
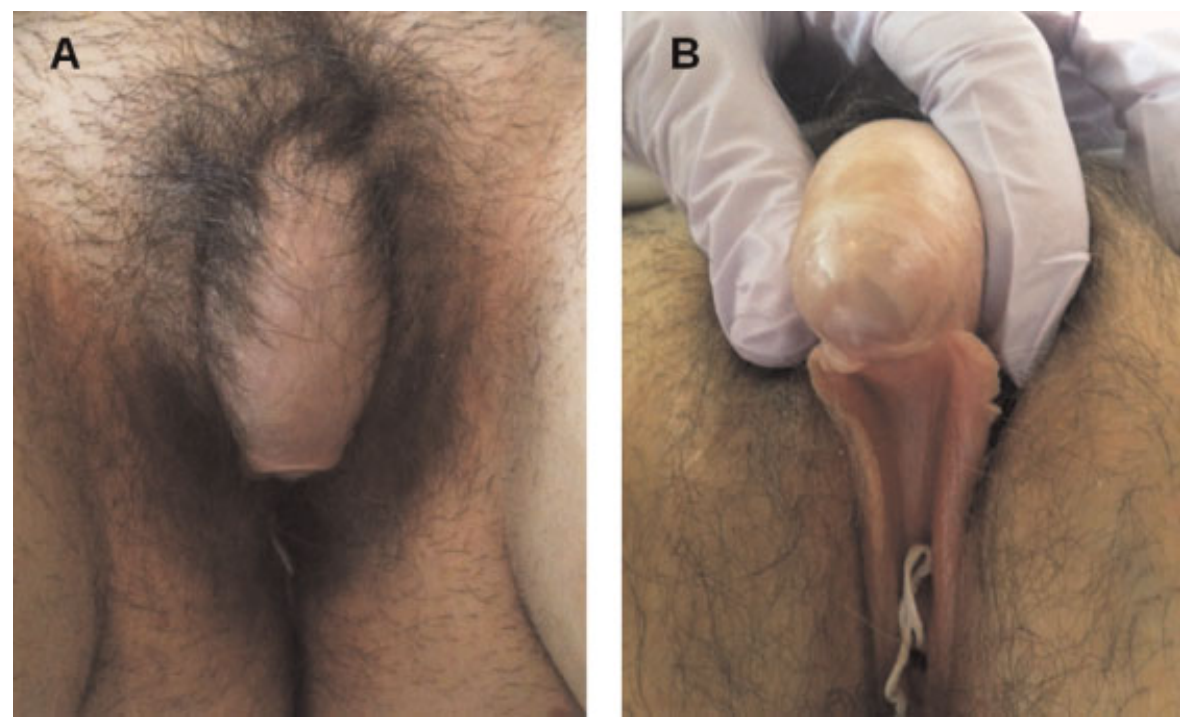

Fig. 1 External genital exam. 
Table 1 Hormonal Evaluation of Our Patient

\begin{tabular}{|l|l|l|}
\hline Test & Result & Reference range \\
\hline Plasma ACTH $(\mathrm{pg} / \mathrm{mL})$ & 10 & $6-50$ \\
\hline Morning cortisol $(\mu \mathrm{g} / \mathrm{dL})$ & 12.2 & $4.3-22.4$ \\
\hline Serum albumin $(\mathrm{g} / \mathrm{dL})$ & 4.3 & $3.6-5.1$ \\
\hline SHBG $(\mathrm{nmol} / \mathrm{L})$ & 186 & $1-124$ normal, likely higher in pregnancy \\
\hline $17-O H$ progesterone $(\mathrm{ng} / \mathrm{dL})$ & 193 & $78-457$ pregnancy first trimester \\
\hline Androstenedione $(\mathrm{ng} / \mathrm{dL})$ & 135 & $30-235$ luteal phase, likely higher in pregnancy \\
\hline $11-$ deoxycortisol $(\mathrm{ng} / \mathrm{dL})$ & 34 & $<51$ normal, likely higher in pregnancy \\
\hline Total testosterone $(\mathrm{ng} / \mathrm{dL})$ & 45 & $2-45$ normal, likely higher in pregnancy \\
\hline Free testosterone $(\mathrm{pg} / \mathrm{mL})$ & 1.2 & $0.2-5.0$ normal, likely higher in pregnancy \\
\hline $17-O H$ pregnenolone $(\mathrm{ng} / \mathrm{dL})$ & 46 & $<905$ premenopausal women \\
\hline DHEA sulfate $(\mu \mathrm{g} / \mathrm{dL})$ & 203 & $40-325$ normal, likely higher in pregnancy \\
\hline
\end{tabular}

Abbreviations: 17-OH, 17-hydroxyprogesterone; ACTH, adrenocorticotropic hormone; DHEA, Dehydroepiandrosterone; SHBG, sex hormone-binding globulin.

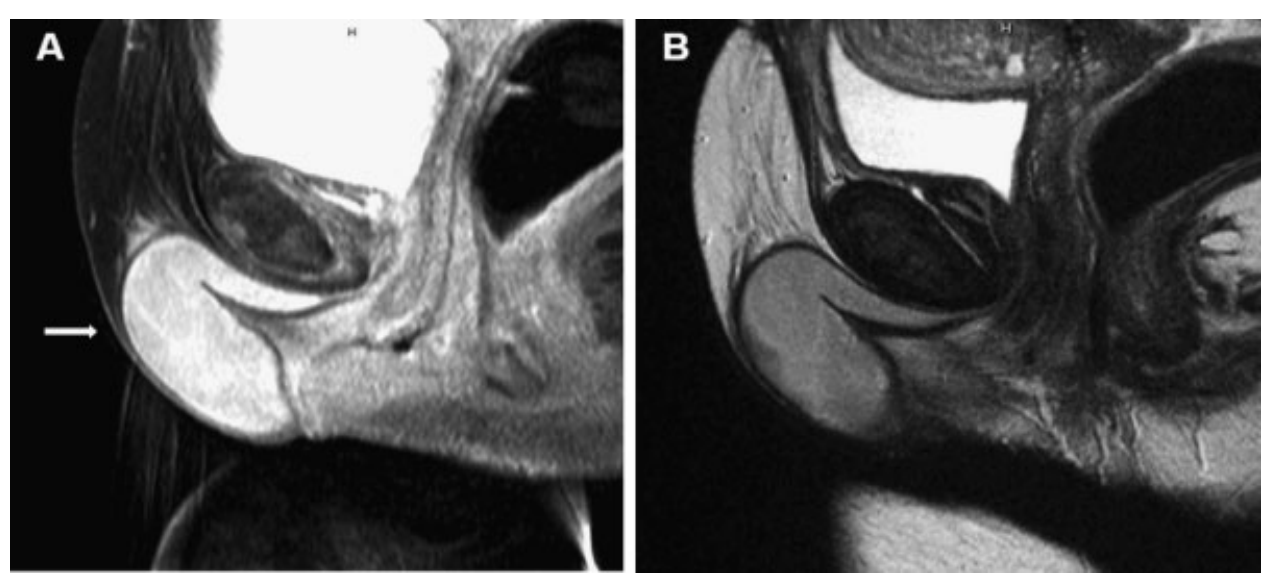

Fig. 2 Magnetic resonance imaging of the pelvis. (A) Sagittal image of the pelvis showing intense T1 hyperintensity within a large $6.2 \times 4.2-\mathrm{cm}$ cyst (arrow), which extends cephalad into the anterior periurethral space. (B) Mild hyperintensity on a T2-weighted sagittal image, corresponding to the proteinaceous fluid-filled cyst.
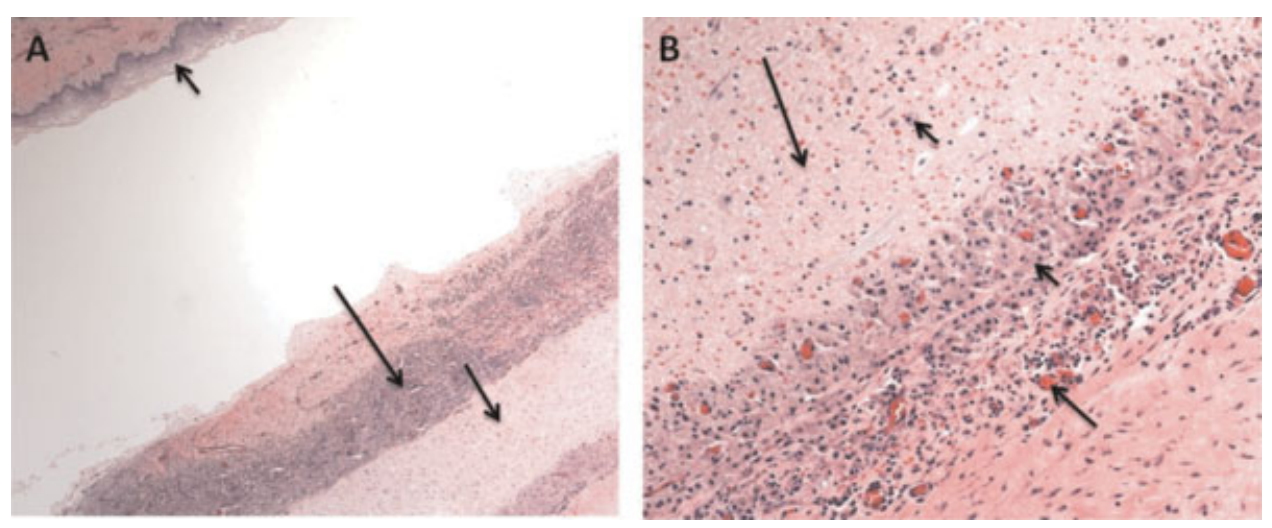

Fig. 3 Histopathology of surgical specimen. (A) Characteristic features of a ruptured epithelial inclusion cyst are shown: squamous epithelial lining (short arrow), necrotic debris (medium arrow), and inflammatory cells (long arrow). Hematoxylin and eosin, $5 \times$ magnification. (B) Macrophages (short arrows) consuming necrotic debris (long arrow) are characteristic of ruptured epidermal inclusion cysts. Neovascularization (medium arrow) is consistent with granulation tissue changes. Hematoxylin and eosin, $20 \times$ magnification. 
CK20 staining, ${ }^{3,4}$ respectively, both of which turned out negative. Postoperatively, the patient had no difficulties from pain, infection, dysuria, or any dyspareunia-in fact, she conceived again at 2 months after the surgery. She is currently in her second trimester, doing well, and has not reported any changes of her clitoral size or any bladder dysfunction. A detailed fetal anatomical survey by ultrasound revealed a female fetus, with measurements consistent with established dates and normal-appearing urogenital structures.

\section{Discussion}

The differential diagnoses for adult-onset clitoromegaly are divided into hormonal and nonhormonal etiologies (-Table 2). The most pronounced cases of clitoromegaly tend to result from hormonally active virilizing tumors, usually of ovarian origin, including Leydig-cell tumors, granulosa cell tumors, thecomas, and sex-cord stromal tumors, which can present with markedly elevated testosterone levels near normal-male range, are fast-growing, and correlate with rapid development of symptoms often over the course of a few months. ${ }^{5}$ Occasionally, adrenal tumors such as adrenocortical carcinomas or adenomas can also lead to clitoromegaly. ${ }^{6,7}$ More commonly, however, patients presenting with ambiguous genitalia are found to have late-onset congenital adrenal hyperplasia, or $\mathrm{CAH}^{8}{ }^{8}$ including deficiencies in 21-hydroxylase, 3- $\beta$-hydroxysteroid dehydrogenase, 11- $\beta$ hydroxylase, and 17- $\alpha$ hydroxylase. In contrast to classical $\mathrm{CAH}$ where virilized genitals are present at birth, late-onset $\mathrm{CAH}$ is diagnosed in adulthood and tends to be milder, presenting with hirsutism, acne, and irregular menses. However, clitoromegaly to the degree observed in our patient would not likely be attributed to $\mathrm{CAH}$, especially given her lack of hirsutism and acne, menstrual irregularities, or infertility. Biochemically, CAH can present as insufficient glucocorticoid production and excess synthesis of mineralocorticoid precursors. However, our patient had no history of hypertension or hypokalemia. Although her first child was a boy, who is healthy and well, developing normally, and without any significant medical history, we did not know the gender of her second fetus and therefore had to evaluate for these conditions, as maternal hyperandrogenism can predispose a female fetus toward virilization, and empiric treatment using dexamethasone early in pregnancy, prior to completion of development of the fetal external genitalia, is often considered when the risk for an affected fetus is high. ${ }^{1}$

Clitoromegaly specifically seen during pregnancy encompasses an interesting group of conditions including luteoma, hyperreactio luteinalis, and placental aromatase deficiency, all of which lead to a transient maternal hyperandrogenism. Luteomas are formed by solid proliferation of luteinized stromal cells under the influence of human chorionic gonadotropin (hCG) during pregnancy and is typically seen at or near term. ${ }^{9,10}$ In contrast, hyperreactio luteinalis can occur at any time during pregnancy and is marked by ovarian enlargement due to theca lutein cysts. ${ }^{11}$ Both of these conditions can involve either unilateral or bilateral ovaries, and although nonneoplastic and usually self-limited, clinically they can mimic ovarian neoplasms and cause local symptoms, therefore biopsy or surgery is sometimes performed for diagnostic and therapeutic purposes. The development of bilateral enlarged ovaries and high hCG levels are also thought to be linked with imbalance of angiogenic factors, which can lead to preeclampsia. ${ }^{12}$ Placental aromatase deficiency is a defect in conversion of androgen to estrogen, which is a process that normally protects the fetus against virilizing androgens. A deficiency in aromatase can cause hyperandrogenemia, leading to maternal virilization in the third trimester as well as pseudohermaphroditism in affected female fetuses. ${ }^{13}$ We speculated that our patient's first pregnancy could have hormonally "primed" the patient and therefore served as a trigger for an extremely rare case of clitoromegaly, but we ultimately abandoned this hypothesis based on the early presentation of her clitoromegaly in relation to the pregnancy, as well as lack of systemic hyperandrogenic cues.

The waxing and waning course of our patient's clitoromegaly and its apparent manifestation during her first pregnancy were baffling, especially considering her normal ambient hormone levels during the course of our evaluation. These

Table 2 Differential Diagnoses of Acquired Clitoromegaly

\begin{tabular}{|l|l|}
\hline Hormonal causes & Nonhormonal causes \\
\hline Late-onset congenital adrenal hyperplasia & Neurofibromatosis \\
\hline Polycystic ovary syndrome & Cysts \\
\hline Virilizing ovarian or adrenal tumors & Female genital cutting, trauma \\
\hline Cushing's disease or syndrome & Hypertrophy due to excess masturbation \\
\hline Synthetic androgen exposure & Beckwith-Wiedemann syndrome \\
\hline Pregnancy-related: & Vulvar tumors, primary or metastatic \\
\hline Placental aromatase deficiency & Cavernous hemangiomas \\
\hline Luteoma & Diverticuli \\
\hline Hyperreactio luteinalis & Abscesses \\
\hline & Fistula \\
\hline
\end{tabular}


data, along with the observation that her virilization appeared exclusively limited to the clitoris, pointed to a local, nonhormonal cause for the clitoromegaly. Pseudoclitoromegaly is an entity that results from mass lesions, such as cysts, diverticuli, abscesses, tumors, or prolapsed genitourinary organs that mimic true clitoral enlargement. Epidermoid or epithelial inclusion cysts are a common type of cutaneous cysts that result from implantation of epidermal elements in the dermis and can occur throughout the body. In the clitoris, it usually occurs as the result of genital mutilation, trauma, or infection and can be painful. ${ }^{14}$ Interestingly, it is thought that such cysts may be able to respond to estrogen stimulation, thereby providing a plausible mechanism for the cyclical nature of the mass. ${ }^{15,16}$ Alternatively, the increased blood flow and pelvic pressure associated with an enlarging gravid uterus provides a conceivable explanation for the pregnancyrelated recurrence of clitoromegaly in our patient.

Surgery is the treatment of choice for symptomatic epidermoid cysts and usually involves marsupialization or enucleation of the mass. These procedures are usually well tolerated and curative. ${ }^{8}$ Complete excision of the cyst wall is desired, as any remnant epithelial tissue may lead to cyst recurrence later.

\section{Conclusion}

Evaluation of adult-onset clitoromegaly requires careful assessment by detailed physical exam, hormonal testing, and, when possible, imaging studies. An urgency in unraveling the underlying diagnosis is of the essence when this phenomenon is identified in pregnancy, as fetal implications of maternal hyperandrogenemia can be devastating. In this case, our patient underwent an expeditious and extensive evaluation to find that this was a nonhormonal process, and she was able to achieve a satisfactory surgical cure of her condition with no detriment to future fertility and reproductive health.

\section{Acknowledgments}

We are gratefully indebted to Dr. Fattaneh Tavassoli of Yale Department of Pathology for discussion of the patient's surgical specimen findings.

\section{References}

1 Speiser PW, et al. A summary of the Endocrine Society Clinical Practice Guidelines on Congenital Adrenal Hyperplasia due to Steroid 21-Hydroxylase Deficiency. Int J Pediatr Endocrinol 2012;2010:494173

2 Androulaki A, Papathomas TG, Alexandrou P, Lazaris AC. Metastatic low-grade endometrial stromal sarcoma of clitoris: report of a case. Int J Gynecol Cancer 2007;17:290-293

3 Sumathi VP, McCluggage WG. CD10 is useful in demonstrating endometrial stroma at ectopic sites and in confirming a diagnosis of endometriosis. J Clin Pathol 2002;55:391-392

4 Al-Khawaja M, Tan PH, MacLennan GT, Lopez-Beltran A, Montironi $\mathrm{R}$, Cheng L. Ureteral endometriosis: clinicopathological and immunohistochemical study of 7 cases. Hum Pathol 2008;39:954-959

5 Horejsí J. Acquired clitoral enlargement. Diagnosis and treatment. Ann N Y Acad Sci 1997;816:369-372

6 Chalasani $S$ et al, Metastatic virilizing adrenocortical carcinoma: a rare case of cure with surgery and mitotane therapy. Clin Med Res 2009 Jun;7(1-2):48-57

7 Pardo JM. Virilization of a female infant due to an adrenal tumor. Clin Pediatr (Phila) 1986;25:456-458

8 Pang S. Congenital adrenal hyperplasia. Baillieres Clin Obstet Gynaecol 1997;11:281-306

9 Masarie K, Katz V, Balderston K. Pregnancy luteomas: clinical presentations and management strategies. Obstet Gynecol Surv 2010;65:575-582

10 Niranjala MW, Wijeyaratne CN, Jayalath GKC. Virilization in a pregnancy. Sri Lanka Journal of Diabetes Endocrinology and Metabolism 2012;2:43-45

11 Schnorr JA Jr, Miller H, Davis JR, Hatch K, Seeds J. Hyperreactio luteinalis associated with pregnancy: a case report and review of the literature. Am J Perinatol 1996;13:95-97

12 Masuyama H, Tateishi Y, Matsuda M, Hiramatrsu Y. Hyperreactio luteinalis with both markedly elevated human chorionic gonadotropin levels and an imbalance of angiogenic factors subsequently developed severe early-onset preeclampsia. Fertil Steril 2009;92: e1-e3

13 Shozu M, Akasofu K, Harada T, Kubota Y. A new cause of female pseudohermaphroditism: placental aromatase deficiency. J Clin Endocrinol Metab 1991;72:560-566

14 Paulus YM, Wong AE, Chen B, Jacobson MT. Preputial epidermoid cyst: an atypical case of acquired pseudoclitoromegaly. J Low Genit Tract Dis 2010;14:382-386

15 Celik N, Yalçin S, Güçer S, Karnak I. Clitoral epidermoid cyst secondary to blunt trauma in a 9-year-old child. Turk J Pediatr 2011;53:108-110

16 Rizk DEE, Mohammed KH, Joshi SU, Al-Shabani AY, Bossmar TR. A large clitoral epidermoid inclusion cyst first presenting in adulthood following childhood circumcision. J Obstet Gynaecol 2007;27:445-448 
